NIST

PUBLICATIONS

Proposed Standard

Practice for Assessing

the Performance of

Gas-Phase Air Cleaning

Equipment

\section{Samuel Silberstein}

U.S. DEPARTMENT OF COMMERCE Natlonal Instltute of Standards and Tochnology

Bullding and Fire Research Laboratory Galthersburg, MD 20899
U.S. DEPARTMENT OF COMMERCE

Robert A Mosbacher, Secretary NATONAL INSTIUTE OF STANDARDS AND TICHNOLOGY

John W. Lyon: Director 



\section{Proposed Standard Practice for Assessing the Performance of Gas-Phase Air Cleaning Equipment}

\section{Samuel Silberstein}

U.S. DEPARTMENT OF COMMERCE Natlonal Institute of Standards and Tochnology

Bullding and Fire Research Laboratory Gaithersburg, MD 20899

March 1991

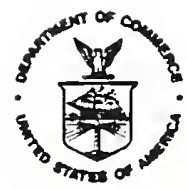

U.S. DEPARTMENT OF COMMERCE Robert A Mosbacher, Secretary NATONAL INSTIUUTE OF STANDARDS AND TECHNOLOGY John W. Lyons, Director 
This proposed standard practice provides a general and flexible laboratory method for assessing the performance of equipment for controlling indoor concentrations of gas-phase air contaminants. Using a canister filled with adsorption media, a profile of breakthrough concentration over time is obtained during each test conducted at a fixed contaminant challenge concentration. Results of tests performed for different contaminants and different challenge concentrations can be used for estimating the useful life of air cleaning equipment, and for comparing equipment. This information will be useful to the engineer for the design and selection of such equipment.

Key words: activated carbon; gaseous contaminant removal equipment; air cleaning equipment; air contaminant; filter media; sorption. 

PROPOSED STANDARD PRACTICE FOR ASSESSING THE

PERFORMANCE OF GAS-PHASE AIR CLEANING EQUIPMENT

Introduction to Standard Practice

The purpose of this paper is to recommended a standard practice for assessing the performance of equipment for reducing indoor concentrations of gas-phase air contaminants. This section presents background information, focusing on the development of the modeling used to describe sorption in air cleaning equipment.

\section{Background}

Need. Air cleaning equipment containing adsorption media such as activated carbon, chemically treated alumina, and other granular sorbents are used to purify indoor air of gas-phase contaminants. These devices can be incorporated into heating, ventilation and air conditioning (HVAC) systems of buildings in a manner similar to particulate control equipment. American Society of Heating, Refrigerating and AirConditioning Engineers (ASHRAE) Standard 52.1-91 (Ref 1) can be used to evaluate particulate filters. There are procedures for evaluating adsorption media for specialized applications, for example removal of radioactive iodine (Ref 2 ), carbon tetrachloride as a measure of activated carbon pore volume ( $\operatorname{Ref} 3$ ),

chlorofluorocarbons ( $\operatorname{Ref} 4$ ), and several highly toxic gases ( $\operatorname{Ref} 5$ ). These methods specify the challenge contaminant, concentration, temperature, $\mathrm{RH}$, and end point of the test.

A general and flexible test method is needed for evaluating the ability of a variety of gas-phase air cleaning equipment to lower the concentration of a wide range of contaminants under different conditions of temperature, $\mathrm{RH}$, and face velocity. The method must be suitable for estimating the useful service life of devices, and for comparing devices. Ideally, only a small number of laboratory measurements should be required to predict performance for a wide range of contaminants, challenge concentration, face velocity, temperature and RH.

Laboratory methods are valuable because they provide controlled conditions, and cost less than field methods.

Rivers ( $\operatorname{Ref} 6$ ) and Ostojic (Ref 7) summarized the difficulties of testing gasphase air cleaning equipment. Typically, a space may contain many unidentified contaminants at low concentrations, that is in the $\mathrm{ppb}$ and $\mathrm{ppm}$ range. It is impractical to test all potentially occurring contaminants, especially since low concentrations require long tests. One approach to decrease the time required for a test is accelerated loading, in which the challenge concentration is raised to load the media, and then lowered for testing. Complications include the necessity of scaling background contaminants, especially water, during accelerated loading. Practically, it is not possible to scale water. For example, suppose that the challenge concentration of a contaminant is increased 10 -fold during accelerated loading. Suppose that it is desired to test the air cleaning device at $40 \% \mathrm{RH}$. It is not possible to increase the water vapor concentration during accelerated loading by much more than 2-fold. Desorption of adsorbates when the challenge concentration is reduced makes accelerated loading inapplicable to reversible sorbents.

Performance tests of gas-phase air cleaning equipment place stringent demands on analytical instrumentation. A single pass through relatively new adsorption media typically reduces already low contaminant concentrations by $1-2$ orders of magnitude. In addition, challenge concentration range of at least one order of 
magnitude must often be tested. Frequently, gas analyzers for several contaminants must be available. A good candidate to satisfy these requirements is a gas chromatography (gc). However, a gas chromatograph may not necessarily be capable of measuring all contaminants of interest, and may require different operating conditions and columns for different classes of contaminants. A gas chromatographmass spectrometer ( $g c-m s$ ) may be especially suitable for the task, but is expensive.

History. Several methods were developed to estimate the service life of respirator cartridges containing activated carbon (Ref 8-10), and gas-phase contaminant removal devices in general (Ref 7). One first determines the equilibrium adsorptive capacity of a cartridge using an adsorption isotherm. This requires separate experimental determination of certain terms (Ref 10). The Mecklenburg equation (Ref 8 ), or later, the Wheeler equation (Ref 11-12), was then used to estimate the time to some specific level of breakthrough or penetration (see Ref 8 or 10 for these equations). "Use of the Mecklenburg equation, although surprisingly accurate, is hindered because of its complexity and the general unavailability of many of the experimental constants. The modified Wheeler equation represents a major simplification in the breakthrough time calculation (Ref 8)." Nelson and Correia (Ref 8) show that the results derived using the Mecklenburg and Wheeler equations are similar. Both closely approximated experimental results up to $40 \%$ penetration.

Yoon and Nelson (Ref 10) modeled adsorption in filter cartridges on the basis of gas adsorption kinetics. The model is similar to Langmuir's: adsorbate binds to a limited number of binding sites on the sorbent surface. Let $Q=$ probability of adsorption and $P=$ the probability of breakthrough. Then $P=$ penetration, and $Q=1$ - $P=$ efficiency = proportion of free binding sites on the sorbent surface. Yoon and Nelson assumed that $-\mathrm{dQ} / \mathrm{dt}=\mathrm{k}^{\prime} \cdot \mathrm{P} \cdot \mathrm{Q}$, where $\mathrm{k}^{\prime}$ is a proportionality constant. From previous results ( $\operatorname{Ref} 13$ ), they concluded that $k^{\prime}=k \cdot C \cdot F / W_{\theta}$, where $F=$ flow rate, $W_{e}=$ mass contaminant absorbed per cartridge sorbent, and $\mathrm{k}$ is a dimensionless proportionality constant. The model results in equations for breakthrough time involving easily calculated terms. The breakthrough time, $t$, is linear in $\ln \left(C_{b} /\left(C-C_{b}\right)\right)$, instead of $\ln \left(C_{b} / C\right)$, as in the Mecklenberg and Wheeler equations, where $\mathrm{Cb}=$ contaminant breakthrough concentration and $\mathrm{C}=$ challenge concentration. As a result, accuracy is increased for values of penetration greater than $40 \%$.

Nelson and Harder (Ref 14) observed that the time to $10 \%$ breakthrough was inversely proportional to a power, approximately 0.67 , of the challenge concentration. This relationship makes it possible to predict the breakthrough time of filter cartridges at untested challenge concentrations from measurements using a small number of challenge concentrations. Grubner and Burgess (Ref 9) recommended against using the relationship because "it does not correspond to physical reality." However, Yoon and Nelson (Ref 15) later derived this relationship using principles of chemical kinetics. Assume that a reaction is of order $n$ in a reactant (for example an adsorbate) whose initial concentration is $C$, and of order 0 in the other reactants (for example sorbent binding sites). The reaction halftime (or time to $50 \%$ penetration in the case of adsorption), $\tau$, would then be proportional to $\mathrm{C}^{-(n-1)}$. If adsorption is complicated enough to consist of various order reactions, then $\tau$ would be observed to be proportional to $\mathrm{C}^{-a}$ for some positive a. (For example if reactions of both order 1 and 2 occur, then "a" would take on some value between 0 and 1.) It can then be shown that $t$, the time to any degree of breakthrough, is also proportional to $\mathrm{C}^{-a}$; in fact $t=\mathrm{K}_{\mathrm{wQ}} /\left(\mathrm{F} \cdot \mathrm{C}^{\mathrm{a}}\right)$, where $\mathrm{K}_{\mathrm{wQ}}$ is a proportionality constant. 
Yoon and Nelson's treatment for filter cartridges is adapted here to serve as the basis for a test method for evaluating air cleaning equipment. It is not difficult to test actual cartridges because they are generally small and fairly uniform in size and shape. Air cleaning equipment, on the other hand, varies widely and can be quite large. It may be impractical to test large equipment in the laboratory. Instead, a small canister containing a quantity of absorption media far smaller than used in the equipment is tested. The problem then arises of applying laboratory results to larger equipment. Scaling to a larger cross-sectional area presents little difficulty because scaling the cross-sectional area and the flow rate by the same factor does not change the face velocity, which is what the sorbent experiences. Yoon and Nelson's equation is consistent with this conclusion: $K_{\text {wQ }}$ is proportional to $W_{e}$, which is proportional to $V=A \cdot Z$, where $A=$ cross-sectional area and $Z=$ bed depth. Since $F=A \cdot v$, where $v=$ face velocity, $t$ is proportional to $Z /\left(v \cdot C^{a}\right)$. Although the equation seemingly applies to different values of $Z$, the model was neither developed nor validated for large values of $Z$. Anong other complications, decreased pressure and face velocity may be significant, and it may be difficult to control temperature and $\mathrm{RH}$ in deep beds. For accurate predictions of useful life, it may be desirable to test full-size equipment (Ref 7 ), although this may be more cumbersome and expensive. Small-scale laboratory tests may be better suited for comparing different types of media for use in the same equipment, even in deep sorbent beds.

Several treatments for the effect of temperature and $\mathrm{RH}$ on air cleaner performance have been developed but none have yet been validated sufficiently for incorporation into a recommended practice (Ref 16-18).

Several single-pass, laboratory test methods have been used to evaluate specific aspects of activated carbon performance, as indicated above (Ref 2-5). These methods were adapted at NIST for flexible testing of adsorption media and challenge contaminants under various conditions (Ref 19-20). A canister for testing media (Ref 2) was incorporated into the NIST test method for reproducibility. A gas chromatograph-flame ionization detector (gc-fid) was used with and without a methanizer, depending on the test contaminant.

A public design review meeting was held at NIST in 1988 (Ref 21) to evaluate the test method. Participants agreed that the gas-phase contaminant removal efficiency of adsorption media can be measured under laboratory conditions using the method developed at NIST. The test conditions should be consistent with typical field use insofar as possible. Gas-phase contaminants representing a wide variety of chemical species and concentrations should be tested if the exact target contaminants cannot be specified in advance. It was suggested that the following be tested: toluene ( 1 to $100 \mathrm{ppm}$ ); carbon monoxide (100 and $10 \mathrm{ppm}$ ); formaldehyde ( 1 and $0.1 \mathrm{ppm}) ; \mathrm{NO}_{2}(0.3$ and $0.03 \mathrm{ppm})$; Methyl isobutyl ketone (10 and $\left.1 \mathrm{ppm}\right)$; and butylcellosolve (100 and $10 \mathrm{pprn}$ ).

The test method developed at NIST (Ref 19) took into account channeling, that is, the imperfect packing of granular adsorption media within canisters that permitted a small fraction of air to effectively bypass the media. Mahajan reports that the mass of the granular media varied by up to 48 even when canisters were filled according to a standard protocol. Nelson and Correia (Ref 8), however, state that channeling rarely arises from incompletely filled cartridges. The present recommended practice does not treat flow bypass within the canister because it would complicate and lengthen the tests to account for an effect of unknown importance in the field. 


\section{Standard Practice}

1 Purpose. The recommended practice is a general and flexible laboratory method for assessing the performance of air cleaning equipment for controlling indoor concentrations of gas-phase air contaminants. A profile of breakthrough concentration or penetration over time is obtained during each test conducted at a fixed contaminant challenge concentration. Results of tests conducted for different contaminants and different challenge concentrations can be used for estimating the useful life of air cleaning equipment adsorption media, and for comparing equipment. This information will be useful to the engineer for the design and selection of such equipment.

2 Classification. Gas-phase air cleaning equipment is classified by whether the adsorption media bind contaminants reversibly or irreversibly. Reversible sorbents, notably activated carbon, at equilibrium with a challenge concentration, desorb adsorbates if the challenge concentration is reduced. (More precisely, there is net desorption. Sorption is a dynamic process with adsorption and desorption occurring simultaneously. Only net adsorption or desorption is observed on a bulk scale depending on whether the challenge concentration is large or small.) Irreversible sorbents may contain catalysts that chemically bind or transform adsorbates so that they are not desorbed when the challenge, or inlet, concentration is reduced.

For reversible sorbents, one must determine whether desorption results in tolerable downstream contaminant concentrations. This is not a concern for irreversible sorbents; their potential problem is release of toxic effluents from the chemical reaction undergone by adsorbates.

Testing may be considerably shortened by loading irreversible sorbents with a contaminant at a high concentrations ("accelerated loading") until just before the contaminant penetration reaches the level of interest.

3 Scope. This practice recommends suitable methods for assessing the contaminant removal efficiency of gas-phase air cleaning equipment as installed in laboratory test airstreams and challenged with prepared gases. Only the chemical and physical components of adsorption media testing are treated here, not the equally important engineering (pressure loss) and economic aspects.

Because equipment can be used under varied conditions and the recommended practice is intended to be general, it cannot specify challenge contaminants, their concentration, analytical methods for measuring concentrations, and the temperature and $\mathrm{RH}$ during conditioning and testing. Appropriate contaminants, test conditions, and monitoring devices must be selected by the user on the basis of the anticipated conditions of use of air cleaning equipment. Straightforward calculations are used to generalize the test results to different challenge concentrations, air face velocity, and media bed depth. Present models for predicting media performance at different values of temperature and $\mathrm{RH}$ have not yet been sufficiently validated.

The user must also establish acceptable performance criteria. For example, the following criteria as well as others may be given varying weight: time to $5 \%$ or 10 of penetration at particular challenge concentration ranges of specific contaminants, performance over a particular range of temperature and $\mathrm{RH}$, and performance in the presence of high or low concentrations of specified background contaminants. Section 5.4 provides several quality assurance checks to serve as a basis for defining acceptable performance. 
The linear model for breakthrough time as a function of penetration is likely to fail at high values of $\mathrm{RH}$, generally above about 60\% (Ref 16-17), in combination with high penetration, generally above about 908 . Since penetration of the order of 108 or less is usually of interest, this should not be a severe limitation.

\section{Definitions}

absorption: a process whereby a porous material extracts one or more substances from an atmosphere, a mixture of gases, or a mixture of liquids ( $\operatorname{Ref} 22$ ).

accelerated adsorption tests: adsorption tests in which the end point is hastened by testing at conditions more severe than those anticipated in service (Ref 23).

accelerated service life: the elapsed time until the end point is reached in an accelerated adsorption test ( $\operatorname{Ref} 23$ ).

activated carbon: a form of carbon made porous by special treatment so it is capable of adsorbing various odors, anesthetics, and other vapors ( $\operatorname{Ref} 22$ ).

activity: a generic term used to describe the capacity to adsorb in general; also, the adsorptive capacity of an adsorbent as measured by a standard test (Ref 23).

adsorbate: any substance that is or can be adsorbed ( $\operatorname{Ref} 23$ ).

adsorbent: any solid having the ability to concentrate significant quantities of other substances on its surface (Ref 23 ).

adsorption: a process in which fluid molecules are concentrated on a surface by chemical or physical forces, or both (Ref 23).

adsorption media: a granular substance that removes gaseous contaminants from a fluid by adsorption processes.

air contaminant: a substance (solid, liquid, or gaseous) which is not found in the normal composition of the atmosphere (Ref 22).

breakthrough concentration: the contaminant concentration downstream of an air cleaning device.

challenge concentration: the contaminant concentration at the inlet of an air cleaning device.

channeling: the greater flow of fluid through passages of lower resistance which can occur in fixed beds or columns of particles due to nonuniform packing, irregular sizes and shapes of the particles, gas pockets, wall effects, and other causes (Ref 23).

chemisorption (chemical adsorption): the binding of an adsorbate to the surface of a solid by forces whose energy levels approximate those of a chemical bond (Ref 23). 
density, apparent (density, bulk): the mass under specified conditions of a unit volume of a solid sorbent including its pore volume and inter-particle voids (Ref 23).

desorption: the separation of an adsorbate as such from a sorbent (Ref 23).

efficiency: 1 - penetration, often expressed as a percentage.

end point: the occurrence in the effluent of the maximum permissible concentration of an adsorbate of interest ( $\operatorname{Ref} 23$ ).

equilibrium adsorptive capacity: the quantity of a given component adsorbed per unit of adsorbent from a fluid or fluid mixture at equilibrium temperature and concentration, or pressure (Ref 23).

face velocity: air flow rate divided by the cross-sectional area of the adsorption media bed perpendicular to the flow.

granular activated carbon: activated carbon in particle sizes predominantly greater than 80 mesh (Ref 23).

half-time: the time required for a reactant concentration to reach one-half the difference between the initial and background concentration.

irreversible adsorption: adsorption in which the desorption isotherm is displaced toward higher equilibrium adsorption capacities from the adsorption isotherm (Ref 23).

isotherm: a plot of quantity adsorbed per unit of adsorbent against equilibrium concentration, or pressure, when temperature is held constant (Ref 23).

moisture content: the water content of a substance as measured under specified conditions ( $\operatorname{Ref} 23$ ).

penetration: the ratio of breakthrough to challenge concentration, often expressed as a percentage.

physical adsorption (van der Waals adsorption): the binding of an adsorbate to the surface of a solid by forces whose energy levels approximate those of condensation $(\operatorname{Ref} 23)$.

pore volume: volume of the pores in a unit weight of a sorbent (Ref 23).

powdered activated carbon: activated carbon in particle sizes predominantly smaller than 80 mesh ( $\operatorname{Ref} 23$ ).

preferential adsorption: adsorption in which a certain component or certain components are adsorbed to a much greater extent than others (Ref 23 ).

reversible adsorption: adsorption in which the desorption isotherm approximates the adsorption isotherm ( $\operatorname{Ref} 23$ ). 
service life (service time): the elapsed time until the end point in reached in an adsorption process (Ref 23).

sorption: a general term covering both absorption and adsorption (Ref 22).

\section{Recommended Practice}

\subsection{Instruments and Materials}

air: compressor used to supply compressed air should be of the oil-free type to minimize injection of hydrocarbons. It must be capable of pressurizing air to at least $300 \mathrm{kPa}$.

balance: capable of mass determination with accuracy and precision of $10 \mathrm{mg}$.

canister: shown in Fig. 1; described in Section 6.2 of Ref 2 .

concentration monitor: must measure challenge air contaminant concentrations at or near real-time, but at least every 15 minutes, to a relative precision and accuracy of 58 or better. Concentrations downstream of the filter media should be measured to a relative precision and accuracy of $20 \%$ or better.

contaminant supply system: must emit pure contaminant gases into the supply airstream, with relative accuracy and precision of injection rate better than $5 \%$.

data acquisition system: must be capable of automatically recording time, temperature and $\mathrm{RH}$ of the airstream, and challenge and purified air contaminant concentration.

dew point hygrometer: must measure dew point of the inlet airstream to relative accuracy and precision within $0.5 \%$.

distilled water.

humidification system: should maintain $\mathrm{RH}$ of the airstream within 5 of the desired value.

feed funnel and vibrator: in accordance with the Procedure Section of ASTM Test Method D2854 ( $\operatorname{Ref} 24$ ).

flow measurement and control: mass flow controllers, control valve and orifice meter, rotameter or any other device with adequate stability and demonstrated measurement system precision and accuracy of 28 of specified flow rate at the test conditions. No flow measuring device should be located directly downstream of the test bed such that it is subject to variable temperature and $\mathrm{RH}$ during a test as a result of water absorption by the carbon.

fume hood: Many of the contaminants, carrier gases, and reagents likely to be used in the test method are hazardous, explosive, or may constitute a fire hazard.

Either the tests should be conducted under a fume hood, or hazardous gases should be vented into a fume hood. 
pressure regulator.

riffle sampler: in accordance with 35.5 .2 of American Society of Testing and Materials (ASTM) Practice E300.

temperature measurement: device must measure airstream temperature with precision and accuracy of $0.2^{\circ} \mathrm{C}$.

thermostat: must maintain ambient air temperature within $1^{\circ} \mathrm{C}$ of the setpoint.

\subsubsection{Calibration}

Maintain calibration of thermometers, flowmeters, flow controllers, regulators, and balances by standard laboratory methods.

For each contaminant, calibrate the gas analyzer over the range of concentrations to be measured. Use at least three concentrations spanning an approximately 100fold concentration range prepared from a calibration standard traceable to NIST.

\subsection{Apparatus. Any testing scheme providing for the proper face velocity,} contaminant concentration, air and contaminant purity, temperature and RH control, and analytical capability may be used for testing gas-phase air cleaning media. The scheme used at NIST (Ref 19) is shown in Fig. 2 and described below. Other schemes may be more suitable to particular applications. For example, a method (Ref 2) designed to simulate conditions of steam leakage in nuclear reactors, where RH may be as high as 95\%, is, by necessity, far more elaborate than the method described here.

Compressed laboratory air at a pressure of about $350 \mathrm{kPa}$ (PRl in Fig. 2) at room temperature, is dried through silica gel, and purified through activated carbon, molecular sieve, potassium permanganate-impregnated alumina, or other agents ("pre-filter").

The airstream is split into two variable sized components. One component is humidified by passing through a 25-L polyethylene bottle filled with 15-20 L of distilled water ("humidification section"). Air enters the bottle through a finely perforated nylon tube, is bubbled through water, passes through a restrainer, and leaves from the top of the vessel. The water temperature is measured by a platinum resistance temperature detector (RTD). The water temperature is maintained constant by placing the bottle in an insulated container or by controlling the room temperature to a constant level. The large volume of water also helps maintain constant temperature. The two airstreams are combined; their relative flow rates determine RH. An alternative way to control RH is to chill or heat the water rather than to split and recombine the airstream. In this case, the bottle must be insulated; the airstream returns to room temperature in the tubing after leaving the bottle. The temperature and RH of the airstream are measured with a thermistor and a dew point hygrometer (labelled "dew point temperature" in Fig. 2), respectively. Gas-phase contaminant is introduced into the airstream through port PO at a constant flow rate (FR2 and FM2) large enough to maintain the desired inlet concentration. Contaminants may be supplied from compressed bottled gases of sufficient purity, from permeation tubes, or other sources.

The canister assembly shown in Fig. 3 consists of cylindrical vessels, top and bottom end caps, and four test canisters. Contaminated air enters the canister 
assembly through the side wall, where the air flow expands as well as changes its direction by $90^{\circ}$, enhancing mixing. Air is sampled through ports P1 and P2 (Fig. 2) at regular intervals and directed to a gas analyzer (for example, a gc in Fig. 2) where the inlet and outlet concentrations are measured. (Strictly speaking, the inlet concentration does not have to be measured if it is known by other means, for example from the contaminant generation rate and the air entry rate. In that case, it may be measured for quality assurance.) The open end of the tube of the bottom end cap containing port $\mathrm{P} 2$ is connected to a rotameter type flowmeter (FR1 in Fig. 2) for monitoring the air flow rate through the test apparatus. Air is exhausted through a laboratory fume hood.

The stainless steel canister described in ASTM method D3803 (Ref 2) is shown in Fig. 1. The canister is a $50-\mathrm{mm} I D, 25-\mathrm{mm}$ or $50-\mathrm{mm}$ long, cylindrical vessel. Both ends of the canister are designed to hold a perforated screen, a baffle ring, and a snap ring at each end. One of the ends also has an 0-ring. The space between the two perforated screens is packed with adsorption media. The face velocity should be set as close as possible to the face velocity anticipated under actual use conditions.

5.3 Procedure. This section describes the test procedure for each combination of adsorption media, challenge contaminant, challenge concentration, temperature, RH, face velocity, background contamination, and conditioning protocol.

Step 1 Determine by appropriate means whether media removes contaminants reversibly or irreversibly at the highest challenge concentration to be tested. This can be done by an adsorption followed by a desorption test, for example (described below in this section).

Step 2 Supply purified, conditioned, compressed air to the test apparatus . Adjust the air flow rate through the canister to obtain a constant face velocity close to that expected under actual use.

Step 3 Check the test loop for leaks by passing air through an empty test canister. Inject contaminant into the airstream at a constant rate. The upstream and downstream contaminant concentration should be the same since there is no adsorption.

Step 4 Maintain constant ambient temperature.

Step 5 Condition adsorption media in clean air at fixed temperature and $\mathrm{RH}$ according to a protocol selected by the user. It is crucial that conditioning be done reproducibly, and that the exact conditions be noted because adsorption media service life is strongly dependent on the conditioning protocol, especially $\mathrm{RH}$ (Ref 16).

Step 6 Fill canister with granular adsorption media as described in ASTM Practice E300 (Ref 21). Determine mass of the media during sampling by subtracting mass of empty cartridge from mass of filled cartridge. Determine media volume to nearest 1 $\mathrm{ml}$. If the information is needed, measure the particle size distribution of the media, according to ASTM Test Method D2862 (Ref 26), and the moisture content according to ASTM Test Method D2867 (Ref 27). 
Step 7 Set ambient temperature to the desired test temperature.

Step 8 Apportion air flow through and around the bubbler or adjust the bubbler temperature to obtain the desired test $\mathrm{RH}$ as measured by dew point hygrometer. Equilibrate apparatus for several hours.

Step 9 Place cartridge containing adsorption media into the test loop and inject contaminant into the airstream at a constant rate calculated to produce the desired challenge concentration. To save time, irreversible media may be loaded by alternating intervals of high and low concentrations, measuring the downstream concentration during the latter ("accelerated loading"). The accelerated loading time multiplied by the ratio of the high to the low concentration may be taken as the time it would take to load the air cleaning device using the low concentration.

Step 10 To simulate "dirty" air, purified air may be spiked with a mixture of specific background contaminant gases, in addition to the challenge contaminant. If the composition of the "dirty" air is unknown, then the mixture may be selected to contain the EPA criteria pollutant gases at their regulatory limits (but it is not recommended that lead-containing pollutants be included because of their toxicity). As far as possible, background pollutant concentrations should be increased in proportion to the increase of the challenge concentration during accelerated loading of irreversible sorbents. This may be impossible in the case of RH; for example, there is little room for increasing the $\mathrm{RH}$ beyond a test condition of $50 \%$.

Step 11 Continue test until at least $60 \%$ penetration if this test is to be used to determine the relationship between $C$ and $t$ according to Equations 3-6 below. Otherwise the test may be run to a lower penetration of interest. During tests of catalytic beds, determine any potentially harmful effluents that may be released by the media and measure their concentration.

Step 12 (for reversible sorbents only) After absorption is completed, check whether desorption results in acceptable downstream contaminant concentrations. user must determine what is an "acceptable" concentration.) Use the same air, background contaminants and test conditions for the desorption test as for the adsorption test except for the absence of the challenge contaminant. It may be sufficient to perform this test using only one or a limited number of challenge concentrations. If desorption is acceptable at the highest concentration, it will certainly be tolerable at lower levels. Conversely, if desorption is excessive at a low challenge concentration, it will be even worse at higher concentrations.

5.4 Testing protocol and calculations. This section describes the calculations for characterizing media adsorption. It also outlines the types of tests needed to provide data required for the calculations.

The challenge contaminants, and temperature, $\mathrm{RH}$, and concentration ranges selected for testing should correspond to those expected under actual use of the media. If the conditions under which the adsorption media will be used are uncertain, then challenge contaminants should be selected to represent different classes of chemicals. (The mixture described in the History section is an example.)

It is recommended that test conditions encompass and do not deviate very far from those anticipated in practice. The number of face velocities and challenge concentrations tested should be sufficient to obtain a good estimate of the 
deviation from predicted values. For example, if the challenge concentration of a contaminant is anticipated to be in the range 600-1300 ppb, then a suitable test range might be 500-1500 ppb at intervals of $250 \mathrm{ppb}$.

All of the following calculation procedures are for a single contaminant at the same face velocity, temperature, and $\mathrm{RH}$.

Step 1. For each challenge concentration, fit either breakthrough concentration, penetration, or efficiency to $t$ by linear regression analysis:

$\left.\ln \left(C_{b} /\left(C-C_{b}\right)\right)=\ln (P /(1-P))=\ln ((1-Q) / Q)\right)=-k^{\prime} \cdot(\tau-t)$

Where

$\mathrm{C}_{\mathrm{b}}=$ breakthrough concentration, $\mathrm{mg} / \mathrm{m}^{3}$

$\mathrm{C}=$ challenge concentration, $\mathrm{mg} / \mathrm{m}^{3}$

$\mathrm{P}=\mathrm{C}_{\mathrm{b}} / \mathrm{C}=$ penetration

$\mathrm{Q}=1-\mathrm{P}=$ efficiency

$k^{\prime}=$ rate constant, $\min ^{-1}$

$\tau=$ halftime, min

$t=t$ ime, min

(For clarity, only $P$, and not $C_{b}$ or $Q$ will be used in the remainder of the test method. It is a simple matter for the user to convert to $C_{b}$ or $Q$ if desired.) Calculate $\tau$ as the value of $t$ for which $P=0.5$.

Step 2. Calculate $t$, the time to some specific value of $P$, for example 10\%, as:

$t=\tau+\left(1 / k^{\prime}\right) \cdot \ln (P /(1-P))$

Step 3. Equations $3-5$ permit one to predict adsorption media performance at values of $C$ different from those tested:

$t=K_{m} \cdot m /\left(F \cdot C^{a}\right)=K_{V} \cdot Z /\left(v \cdot C^{a}\right)=K_{V} \cdot V /\left(F \cdot C^{a}\right)$

$F=A \cdot v$

$\mathrm{V}=\mathrm{A} \cdot \mathrm{Z}$

To use Equation 3, first calculate the value of $a$, and $K_{m}$ or $k_{V}$ using linear regression analysis (if 3 pairs ( $t, C$ ) are available; otherwise solve 2 linear equations for the 2 unknowns $\ln K_{m}$ or $\ln K_{v}$, and a):

$\ln t=\ln K_{m}+\ln m-\ln A-\ln v-a \cdot \ln C$

$=\ln \mathrm{K}_{\mathrm{v}}+\ln \mathrm{Z}-\ln \mathrm{v}-\mathrm{a} \cdot \ln \mathrm{C}$

Where

a, $K_{m}$ and $K_{V}$ are linear regression coefficients. $K_{m}$ is used for adsorption per unit mass of media, $K_{V}$ for adsorption per unit volume.

$\mathrm{m}=$ media mass, $\mathrm{g}$

$Z=$ media bed depth, $m$ 
$A=$ cross-sectional area, $\mathrm{m}^{2}$

$\mathrm{v}=$ face velocity, $\mathrm{m} / \mathrm{min}$

$\mathrm{F}=$ air flow rate, $\mathrm{m}^{3} / \mathrm{min}$

$\mathrm{V}=$ bulk media volune, $\mathrm{m}^{3}$

Application of Equation 3 to conditions different from test conditions is described in Section 5.6 below.

Step 4. If desired, calculate $W_{m}$, the mass of adsorbed contaminant per unit mass of adsorption media, or $\mathrm{W}_{\mathrm{V}}$, the mass of adsorbed contaminant per unit volume, for any value of $C$ :

$\mathrm{W}_{\mathrm{m}}=\mathrm{C} \cdot \tau \cdot \mathrm{A} \cdot \mathrm{v} / \mathrm{m}$

$W_{\mathrm{V}}=C \cdot \tau \cdot \mathrm{V} / \mathrm{Z}$

Where

$\mathrm{W}_{\mathrm{m}}=$ mass of contaminant absorbed per unit mass of adsorption media

$W_{\mathrm{V}}=$ mass of contaminant absorbed per unit volune of adsorption media, $\mathrm{g} / \mathrm{m}$

Step 5. Quality assurance. One ensures that the model used here applies to the particular application by using several concentrations and face velocities that encompass the range to be expected. Calculate $\mathrm{k}$ as:

$\mathrm{k}=\tau \cdot \mathrm{k}^{\prime}$

for each value of $C$. The value of $k$ should be constant for all concentrations. Variance in $k$ indicates either deviation from the model or measurement problems. Similarly, the extent of deviation from the lines predicted by linear regression analysis in Equations 1 and 6 is a quality assurance indicator.

As indicated in section 5.2 , two independent challenge concentration determinations serve as a quality assurance check for the contaminant generator and of the gas analyzer.

While there are no adequate ways to accurately predict the value of the exponent "a" in Equation 3 and the dependence of $t$ on temperature at a fixed value of $C$, previous results provide rules of thumb. A marked deviation can indicate a problem in the test system. The exponent "a" has been found to be $0.67 \pm 0.17$ for many contaminants in the concentration range 50-3000 ppm ( Ref 14). Each $10^{\circ} \mathrm{C}$ increase in temperature has been found to result in a $1-108$ relative increase in $t$ for some specific penetration ( $\operatorname{Ref} 16$ ).

Step 6. Repeat steps 1-5 for different combinations of temperature and RH, if necessary. Derive an empirical relationship between $t, W_{m}$ or $W_{V}$ and temperature and $\mathrm{RH}$ at specific values of $\mathrm{C}$ and $\mathrm{v}$.

5.5 Report. Record date, identity of media, media mass and volume, conditioning protocol, run number, media canister bed depth, face velocity through canister, cross-sectional area, airstream temperature, dew point, $\mathrm{RH}$, and detection apparatus, background contaminants and concentrations, and other information essential for repeating each run. For each contaminant record its name, the selected value of $t$, 
$C, P, k^{\prime}, k, W_{m}, W_{v}$, and, if available, the standard deviation or standard error for each quantity as appropriate. A graph of $t$ vs. $C$ or $P$ should be provided. If the results are to be applied to different values of concentration, flow rate, or adsorption media bed size, then report the value of $a$, and either $\mathrm{K}_{\mathrm{m}}$ or $\mathrm{K}_{\mathrm{V}}$ as well.

5.6 Application of results to air cleaning devices. The bed depth, cross-sectional area, flow rate, concentration, ambient temperature and $\mathrm{RH}$ may be different from those used in the tests. Determine the actual values of $m$ and $F, Z$ and $v$, or $V$ and $F$ for the air cleaning device. After $a$, and $k_{m}$ or $K_{v}$ have been determined using Equation 6 , substitute the actual value of $C$ and either $m$ and $F, Z$ and $v$, or $V$ and $F$ into the appropriate form of Equation 3 to predict the value of $t$ for the air cleaning device. It is best to test challenge concentrations and face velocities as close as possible to those that will occur in practice. Interpolation to nearby values using Equation 3 is preferable to extrapolation because the equation will have thus been shown to be valid in the range of interest. Similarly interpolation of empirical equations relating $t$ to temperature and $\mathrm{RH}$ may be used to extend test results to actual conditions.

5.7 Precision and bias. Determination of the precision and bias of the recommended practice requires round-robin testing according to ASTM Practice E691 (Ref 28), which has not been done yet.

\section{References}

1. ASHRAE Standard 52.1-91, Gravimetric and Dust Spot Procedures for Testing Air Cleaning Devices Used in General Ventilation for Removing Particulate Matter.

2. American Society for Testing and Materials Test Method D3803-89, Nuclear-Grade Activated Carbon, pp. 360-376 in 1990 Annual Book of ASTM Standards, Volume 15.01, Philadelphia.

3. American Society for Testing and Materials Test Method D3467, Carbon Tetrachloride Activity of Activated Carbon, pp. 354-356 in 1990 Annual Book of ASTM Standards, Volume 15.01, Philadelphia.

4. American Society for Testing and Materials Test Method D5020, Chlorofluorocarbon Activity of Activated Carbon, pp. 393-395 in 1990 Annual Book of ASTM Standards, Volume 15.01, Philadelphia.

5. Military Specification: Charcoal, Activated, Impregnated, ASC, MIL-C-13724, with amendments to March 5, 1986.

6. R. D. Rivers, Practical Test Requirements for Gaseous Contaminant Removal Equipment, pp. 54-64 in Reference 21.

7. N. Ostojic, Test Method for Gaseous Contaminant Removal Devices, ASHRAE Trans.

91 part 2, 11-31, 1985.

8. G. 0. Nelson, A. N. Correia, Respirator Cartridge Efficiency Studies: VIII. Sumnary and Conclusions, Am. Ind, Hyg. Assoc. J. 37, 514-525, 1976. 
9. O. Grubner, W. A. Burgess, Simplified Description of Adsorption Breakthrough Curves in Air Cleaning and Sampling Devices, Am. Ind. Hyg. Assoc. J. 40, 169-180, 1979.

10. Y. H. Yoon, J. H. Nelson, Application of Gas Adsorption Kinetics--I. A Theoretical Model for Respirator Cartridge Service Life, Am. Ind. Hyg. Assoc. J. 45, 509-516, 1984.

11. L. A. Jonas, J. A. Rehrman, Predictive Equations in Gas Adsorption Kinetics, Carbon 11, 59-64, 1973.

12. L. A. Jonas, J. A. Rehrman, The Rate of Gas Adsorption by Activated Carbon, Carbon 12, 95-101, 1974.

13. G. O. Nelson, C. A. Harder, Respirator Cartridge Efficiency Studies: V. Effects of Solvent Vapor, Am. Ind. Hyg. Assoc. J. 33, 391-410, 1974.

14. G. O. Nelson, C. A. Harder, Respirator Cartridge Efficiency Studies: VI. Effect of Concentration, Am. Ind. Hyg. Assoc. J. 37, 205-215, 1976.

15. Y. H. Yoon, J. H. Nelson, Application of Gas Adsorption Kinetics--II. A Theoretical Model for Respirator Cartridge Service Life and Its Practical Applications, Am. Ind. Hyg. Assoc. J. 45, 517-524, 1984.

16. G. O. Nelson, A. N. Correia, C. A. Harder, Respirator Cartridge Efficiency Studies: VII. Effect of Relative Humidity and Temperature, Am. Ind. Hyg. Assoc. J. $37,280-288,1976$.

17. Y. H. Yoon, J. H. Nelson, A Theoretical Study of the Effect of Humidity on Respirator Cartridge Service Life, Am. Ind. Hyg. Assoc. J. 49, 325-332, 1988.

18. W. Underhill, G. Micarelli, M. Javorsky, Effects of Relative Humidity on Adsorption of Contaminants on Activated Carbon, pp. $42-53$ in $\operatorname{Ref} 21$.

19. B. M. Mahajan, A Method for Measuring the Effectiveness of Gaseous Contaminant Removal Devices Progress Report, National Institute of Standards and Technology Interagency Report 88-3666, Gaithersburg, MD, 1988.

20. B. M. Mahajan, A Method for Measuring the Effectiveness of Gaseous Contaminant Removal Filters, National Institute of Standards and Technology Interagency Report 89-4119, Gaithersburg, MD, 1989.

21. P. E. McNal1, Ed., Proceedings of the Symposium on Gaseous and Vaporous Removal Equipment Test Methods, National Institute of Standards and Technology Interagency Report 88-3716, Gaithersburg, MD, 1986.

22. ASHRAE, Terminology of Heating, Ventilation, Air Conditioning \& Refrigeration, 1986 .

23. American Society of Testing and Materials Standard Definitions D2652-76 (Reapproved 1987), Terms Relating to Activated Carbon, pp. 326-328 in 1990 Annual Book of ASTM Standards, Volume 15.01, Philadelphia. 
24. American Society of Testing and Materials Test Method D2854-89, Apparent Density of Activated Carbon, pp. 329-331 in 1990 Annual Book of ASTM Standards, Volume 15.01, Philadelphia.

25. American Society of Testing and Materials Practice E300-86, Sampling Industrial Chemicals, pp. 331-353 in 1990 Annual Book of ASTM Standards, Volume 15.05, Philadelphia.

26. American Society for Testing and Materials Test Method D2862-82 (Reapproved 1987), Particle Size Distribution of Granular Activated Carbon, pp. 332-333 in 1990 Annual Book of ASTM Standards, Volume 15.01, Philadelphia.

27. American Society for Testing and Materials Test Method D2867-83 (Reapproved 1988), Moisture in Activated Carbon, pp. 335-336 in 1990 Annual Book of ASTM Standards, Volume 15.01, Philadelphia.

28. American Society for Testing and Materials Practice E691-87, Conducting an Interlaboratory Test Study to Determine Precision of a Test Method, pp. 430-449 in 1990 Annual Book of ASTM Standards, Volume 14.02, Philadelphia. 


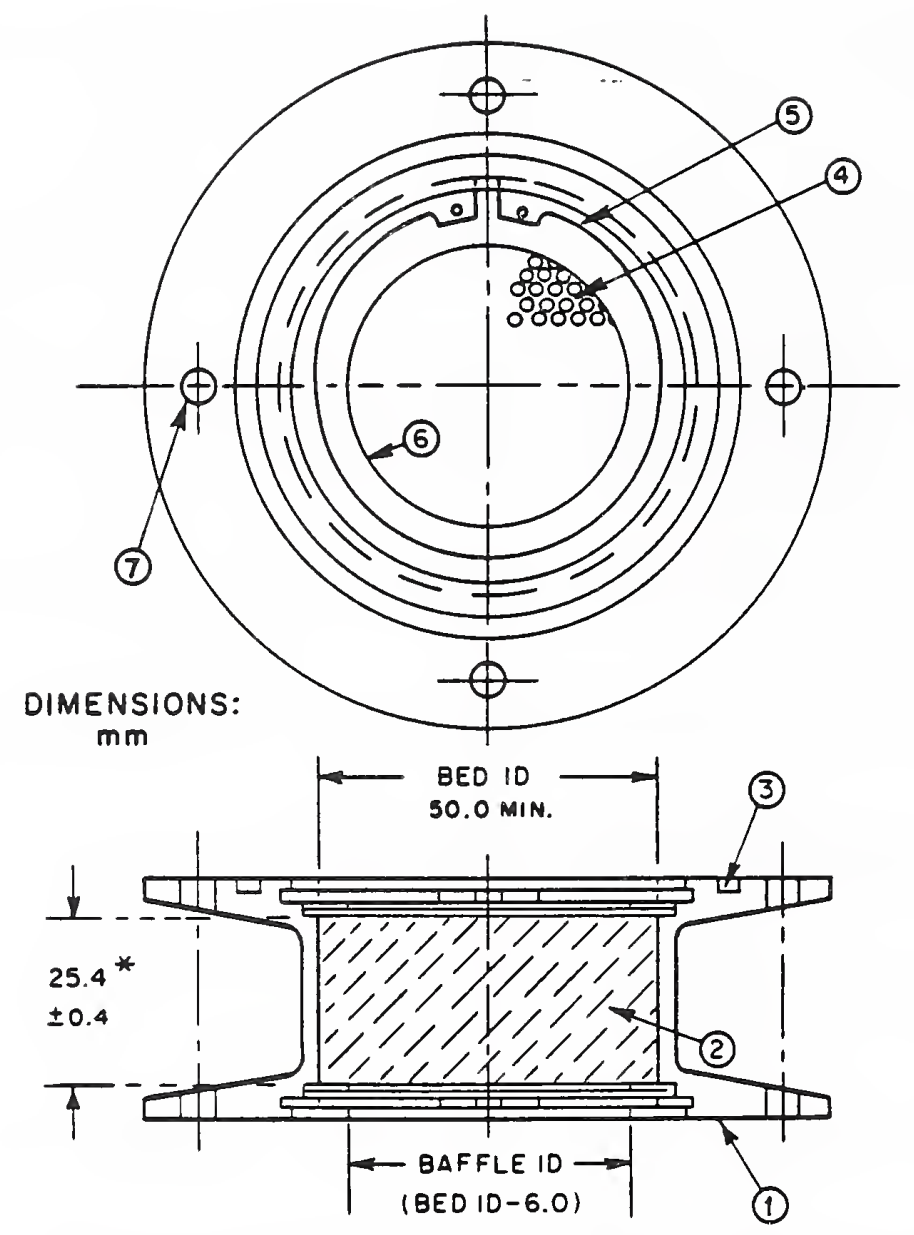

- Standard canister dimension may be used in multiples if desired. Single test canisters of full depth may be used.

\section{1-Bed holder}

2-Adsorption media

3-0-ring gland

4-Perforated screen (both ends)

5-Retaining snap ring (both ends)

6-Baffle (both ends)

7-Holes for assembly tie-rods (four)

Figure 1. Adsorption media test bed holder (canister) (Ref 2). 


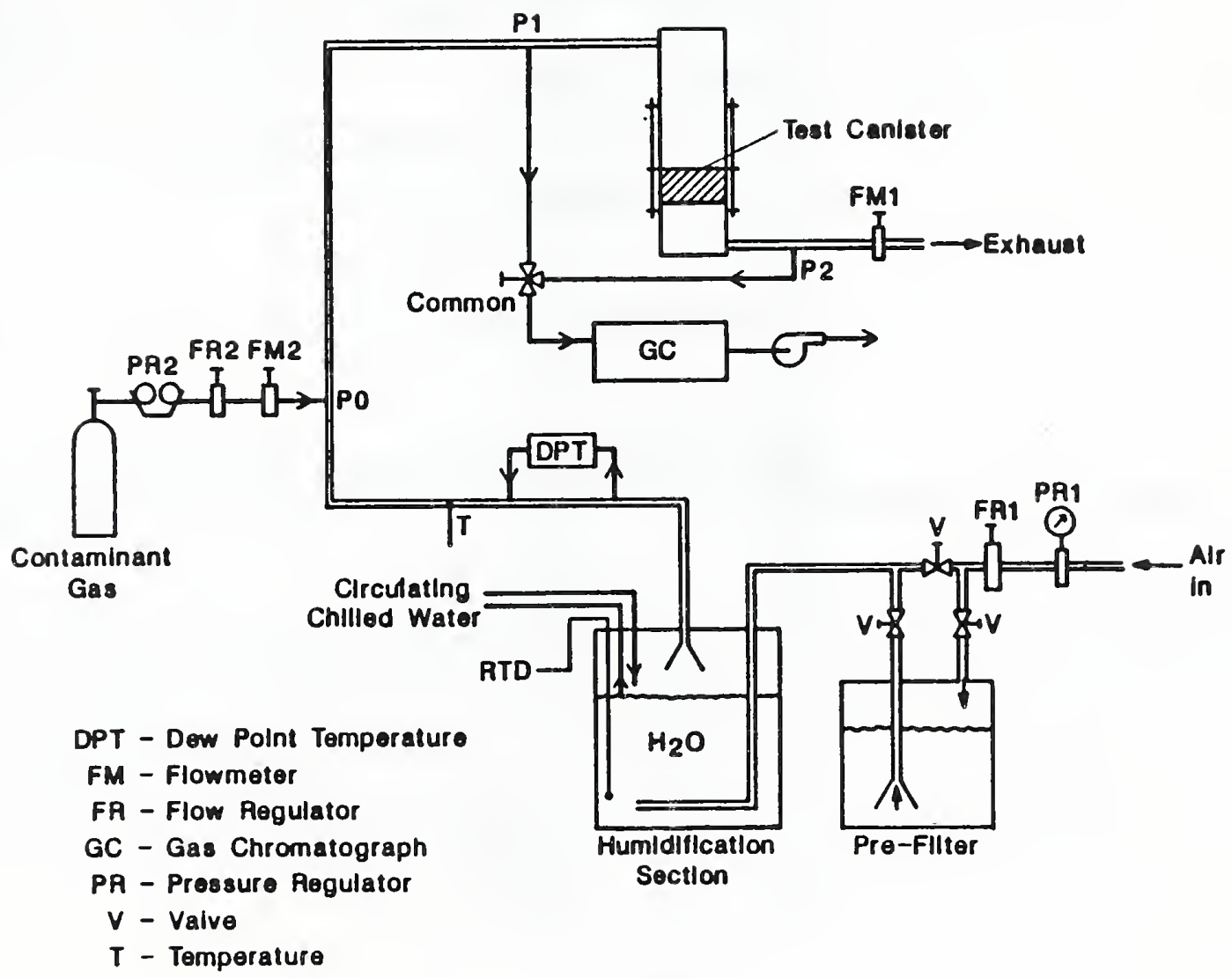

Figure 2. Schematic of test apparatus (Ref 19). The "pre-filter" consists of drying and cleaning agents. $\mathrm{C}=$ contaminant challenge concentration; $\mathrm{C}_{\mathrm{b}}=$ breakthrough concentration. 


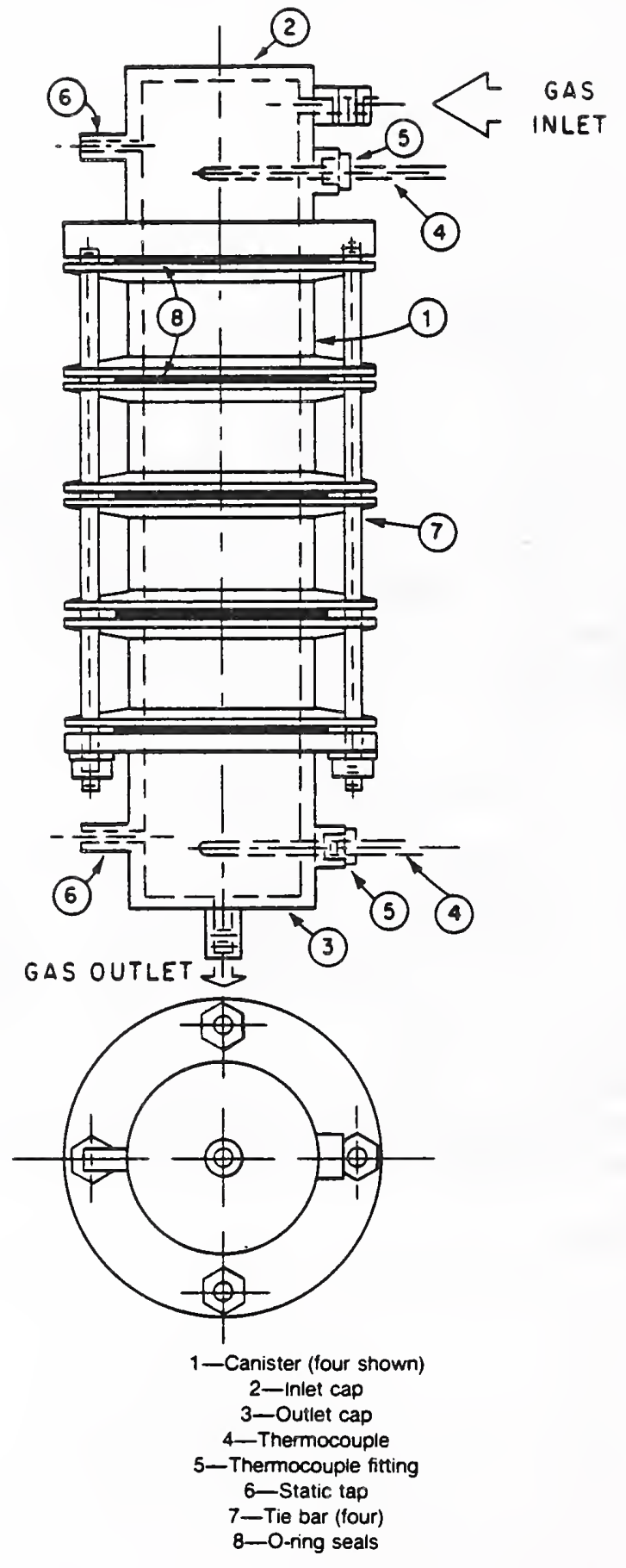

Figure 3. Schematic of canister assembly (test or backup beds) (Ref 2). 


\section{BIBLIOGRAPHIC DATA SHEET}

MARCH 1991

TRLE AND SUBTITLE

Hroposed Standard Practice for Assessing the Performance of Gas-Phase Air Cleaning Equipment AUTHOR(S)

Samuel Silberstein PERFORMINO ORGANIZATION (IF JOINT OR OTHER THAN NIST, SEE INSTRUCTIONS) U.S. DEPARTMENT OF COMMERCE NATIONAL INSTITUTE OF STANDARDS AND TECHNOLOGY GAITHERSBURQ, MD $\approx 0.99$

DOCUMENT DESCRIBES A COMPUTER PROGRAM; SF-185, FIPS SOFTWARE SUMMARY, IS ATTACHED.

ABSTAACT (A 2OO-WORD OR LESS FACTUAL SUMMARY OF MOST SIGNIFICANT INFORMATION. IF DOCUMENT INCLUDES A SIGNIFICANT BIBLOGRAPHY OR LTERATURE SURVEY, MENTION IT HERE.)

This proposed standard practice provides a general and flexible laboratory method for assessing the performance of equipment for controlling indoor concentrations of gas-phase air contaminants. Using a canister filled with adsorption media, a profile of breakthrough concentration over time is obtained during each test conducted at a fixed contaminant challenge concentration. Results of tests performed for different contaminants and different challenge concentrations can be used for estimating the useful life of air cleaning equipment, and for comparing equipment. This information will be useful to the engineer for the design and selection of such equipment.

(WORDS (6 TO 12 ENTRIES; ALPHABETICAL ORDER; CAPITALIZE ONLY PROPER NAMES; ANO SEPARATE KEY WORDS BY SEMICOLONS)

y words: activated carbon; gaseous contaminant removal equipment; air cleaning uipment; air contaminant; filter media; sorption.

FOR OFFICIAL DISTRIBUTION. DO NOT RELEASE TO NATIONAL TECHNICAL INFORMATION SERVICE (NTIS).

ORDER FROM SUPERINTENDENT OF DOCUMENTS, U.S. GOVERNMENT PRINTING OFFICE, WASHINGTON, DC 20402. 

